ShILo, M. (1957). J. gen. Microbiol. 16, 472-481

\title{
The Enzymic Conversion of the Tartaric Acids to Oxaloacetic Acid
}

\author{
By M. SHILO \\ Department of Bacteriology, University of California, Berkeley, \\ California, U.S.A.
}

\begin{abstract}
SUMMARY: Certain bacteria of the genus Pseudomonas attack the tartaric acids by means of inducible stereospecific dehydrases. Each dehydrase converts its specific isomeric substrate to oxaloacetic acid; in crude cell-free extracts the oxaloacetic acid is in turn converted to pyruvic acid, which accumulates. By treatment of the crude extracts with ethylenediaminetetraacetic acid (EDTA), substantial accumulations of oxaloacetic acid can be obtained from the meso- and $d$-tartaric acids, and assays for these two dehydrases, based on the accumulation of oxaloacetic acid in the presence of EDTA, have been developed. This procedure cannot be used to assay the $l$-tartaric acid dehydrase, which is itself very sensitive to EDTA. The patterns of inhibition of the three dehydrases by compounds sterically related to their substrates have been explored, and the findings are interpreted in terms of the minimal steric requirements for enzyme-substrate combination.
\end{abstract}

Many micro-organisms can attack the enantiomorphs of tartaric acid, usually with some degree of steric specificity (Pasteur, 1860, 1863; Barker, 1936; Vaughn, Marsh, Stadtman \& Cantino, 1946; Stadtman, Vaughn \& Marsh, 1945; Tabachnick \& Vaughn, 1948; Mercer \& Vaughn, 1951; Nomura \& Sakaguchi, 1955). Surprisingly, there is only one brief report of microbial attack on meso-tartaric acid (Martin \& Foster, 1955). Until recently, the biochemical mechanisms involved in the metabolism of the tartaric acids have been unknown. Barker (1936) proposed that the dissimilation of $d$-tartaric acid by Aerobacter aerogenes proceeds through oxaloacetate and pyruvate. On the basis of somewhat indirect evidence, the occurrence of this pathway in fluorescent pseudomonads was suggested by Nomura \& Sakaguchi (1955) and by la Rivière (1956). While the work reported here was in progress, it was shown by Krampitz $\&$ Lynen (1956) that the dissimilation of $d$-tartaric acid by an unidentified Gram-positive rod involves a primary dehydration to oxaloacetic acid. A different pathway for the metabolism of the $l$ - and meso-tartaric acids exists in animal mitochondria (Scholefield, 1955; Kun \& Hernandez, 1956; Kun \& Davies, 1956; Kun, 1956). Here, the primary attack occurs by dehydrogenation to oxaloglycollic acid.

This paper describes the enzymic mechanisms of tartaric acid metabolism by a series of Pseudomonas strains, which differ in the specificity of their attack on the three isomers. The existence of strains completely specific for a single isomer greatly facilitated the investigation of this problem. In an accompanying paper (Shilo \& Stanier, 1957), certain physiological properties of the organisms concerned are described. 


\section{Configurational notation}

In the chemical literature, the configurations assigned to the enantiomorphs of tartaric acid are inconsistent: some authors designate the $(+)$-isomer as $\mathrm{L}-(+)$-tartaric acid, others as $\mathrm{D}-(+)$-tartaric acid. As Buchanan (1951) pointed out, this ambiguity is an inevitable consequence of the current configurational notation. From the reference standard, D- $(+)$-glyceraldehyde, one can derive (-)-tartaric acid, via $\mathrm{D}-(-)$-threose; but one can also derive $(+)$-tartaric acid, via D-(-)-glyceric acid, (+)-isoserine and (+)-malic acid. This confused situation can only be remedied by the adoption of a new series of conventions for the specification of configuration, such as those proposed by Cahn \& Ingold (1951). In the meantime, the biologist is faced with a dilemma. We shall resolve it by referring to the tartaric acids and the related substances discussed in this and the ensuing paper in terms of their rotations, as $d$-tartaric acid, $d$-malic acid, etc. The configurational relationships, using the Fischer convention, are indicated by the following structures:

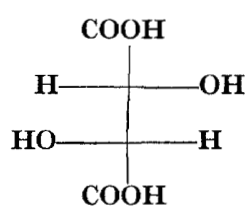

d-tartaric acid, dextrorotatory tartaric acid, natural isomer

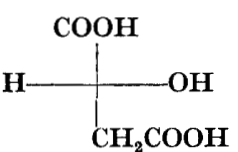

$d$-malic acid, dextrorotatory malic acid, unnatural isomer

\section{METHODS}

Organisms and conditions of cultivation. Pseudomonas strains meso $1, d 15$ and $l 1$ were used. They were isolated by elective culture with meso-, $d$ - and $l$-tartaric acids, respectively. In all cases, the attack on the isomers of tartaric acid was strictly and specifically inducible. Strains meso 1 and $d 15$ can attack only meso- and $d$-tartaric acid, respectively. Strain $l 1$ can be induced to attack either $d$ - or $l$-tartaric acid, by growth in the presence of the appropriate isomer.

The basal medium used for the preparation of specifically induced organisms consisted of: $\mathrm{Na}$ tartrate, $5 \cdot 0 \mathrm{~g}$.; $\mathrm{NH}_{4} \mathrm{Cl}, 1 \mathrm{~g}$.; $\mathrm{MgSO}_{4} .7 \mathrm{H}_{2} \mathrm{O}, 0.05 \mathrm{~g}$. $; \mathrm{CaCl}_{2} \cdot 2 \mathrm{H}_{2} \mathrm{O}$, $0.05 \mathrm{~g}$; $\mathrm{FeCl}_{3}, 0.05 \mathrm{~g}$; Difco yeast extract, $0.025 \mathrm{~g}$; phosphate buffer $(0.01 \mathrm{M}, \mathrm{pH} 7 \cdot 2), 1000 \mathrm{ml}$. Cultures were incubated at $30^{\circ}$ on a mechanical agitator in Fernbach flasks (2 l. capacity) containing $1000 \mathrm{ml}$. medium. Organisms were harvested before termination of the exponential phase of growth.

Chemicals. $d$-Malic acid (the unnatural isomer) and $d$-, $l$ - and meso-isomers of tartaric acid were purchased from the California Foundation for Biochemical Research. The cis and trans isomers of $d l$-epoxysuccinic acid were kindly furnished by Dr J. W. Foster.

Preparation of extracts. The organisms from $1 \mathrm{l}$. of culture medium were

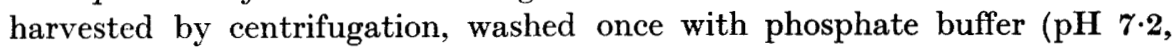


$0.02 \mathrm{M}$ ), and resuspended at a density of $4-5 \%(\mathrm{w} / \mathrm{v})$ in the same buffer. This suspension was then disintegrated by treatment for $10 \mathrm{~min}$. in an ice-cooled Raytheon 9-KC sonic oscillator. Coarse debris and the particulate fraction were sedimented by centrifugation for $30 \mathrm{~min}$. at $19,000 \mathrm{~g}$, yielding a clear straw-coloured supernatant liquid which contained $c .10 \mathrm{mg}$. protein $/ \mathrm{ml}$. Such extracts were used in all the experiments to be described.

Chemical methods. Pyruvic acid and total keto acids were estimated colorimetrically by the method of Friedman \& Haugen (1943), and chromatographically by the method of Cavallini, Frontali \& Toshi (1949). $\beta$-Ketoacids were measured manometrically by catalytic decarboxylation with 4 -aminoantipyrine (Akamatsu, 1950; Sistrom \& Stanier, 1953). Oxaloacetic was determined quantitatively by light absorption at $280 \mathrm{~m} \mu$. with a Beckman model DU spectrophotometer (Green, Leloir \& Nocito, 1945; Cammarata \& Cohen, 1951). $d$ - and $l$-Tartaric acids were estimated colorimetrically by the metavanadate method (Matchett, Legault, Nimmo \& Notter, 1944), and detected chromatographically on Whatman no. 1 paper after development with butanol/water/acetic acid mixture $(50: 40: 10)$ by spraying with bromphenol blue. Protein estimations were made by Pardee's modification of the biuret method (Pardee, personal communication). Manometric measurements of oxygen uptake and $\mathrm{CO}_{2}$ evolution were carried out in the conventional Warburg apparatus at $30^{\circ}$ with air as the gas phase.

\section{RESULTS}

\section{The formation of oxaloacetic acid and pyruvic acid from the tartaric acids}

Crude extracts, centrifuged at gravitational fields insufficient to sediment all the cytochrome-bearing particles, can oxidize specifically the tartaric acid isomers for which the bacteria were induced; however, the very high endogenous respiration makes the data difficult to interpret. When extracts were thoroughly clarified by centrifugation, they showed a very low endogenous oxygen consumption, which was only slightly increased by the addition of tartaric acid. However, with such extracts a rapid evolution of $\mathrm{CO}_{2}$ resulted from the addition of the appropriate isomer of tartaric acid; the total amount of $\mathrm{CO}_{2}$ evolved amounted to slightly more than one mole/mole tartaric acid supplied.

Typical manometric data, obtained with an extract from strain meso 1 acting upon meso-tartaric acid, are shown in Fig. 1. The same type of manometric data was obtained with extracts of strains $d 15$ and $l 1$, acting upon the $d$-and $l$-isomers, respectively. Chromatographic analysis showed that the tartaric acid furnished had disappeared completely from the reaction mixture at the time when the evolution of $\mathrm{CO}_{2}$ had ceased. These observations establish that the primary attack on all three isomers is a non-oxidative one. In each case, chemical analysis of the reaction mixture showed that pyruvic acid had accumulated in appreciable quantities; it was the only major keto-acid present (Table 1).

The formation of $\mathrm{CO}_{2}$ and pyruvic acid from tartaric acid suggested that the 
first product formed might be oxaloacetic acid, which did not accumulate because of its rapid decarboxylation. Accordingly, the effect of ethylenediamine tetraacetic acid (EDTA) on tartrate breakdown was tested. Since EDTA chelates $\mathrm{Mg}^{++}$, it might be expected to inhibit the decarboxylation of oxaloacetic acid.

Table 1. The formation of pyruvic acid from the three isomers of tartaric acid in the presence of specific bacterial extracts

The incubation mixtures contained $100 \mu$ mole sodium tartrate and $0.5 \mathrm{ml}$. of enzyme extract in phosphate buffer $(0.02 \mathrm{M}, \mathrm{pH} 7 \cdot 2)$ in a total volume of $2 \mathrm{ml}$. The mixtures were incubated at $30^{\circ}$ for $10 \mathrm{~min}$., after which the reactions were stopped by adding $2 \mathrm{ml}$. of $10 \%$ trichloroacetic acid. Pyruvate was estimated in the deproteinized supernatant fluid by the Friedman \& Haugen (1943) method, with xylene for extraction of the ketoacid.

\begin{tabular}{|c|c|c|c|}
\hline Substrate & $\begin{array}{c}\text { Source of } \\
\text { extract } \\
\text { (strain) }\end{array}$ & $\begin{array}{c}\text { Pyruvate } \\
\text { found } \\
(\mu \text { mole })\end{array}$ & E 420/E 520 \\
\hline$d$-Tartaric acid & $d 15$ & $12 \cdot 7$ & $\mathbf{1} \cdot \mathbf{3 7}$ \\
\hline$l$-Tartaric acid & $l 1$ & $11 \cdot 6$ & $1 \cdot 38$ \\
\hline meso-Tartaric acid & meso 1 & $26 \cdot 7$ & $1 \cdot 31$ \\
\hline
\end{tabular}

The addition of EDTA (final concentration 0.04 $\mathrm{M}$ ) to an extract from strain meso 1 virtually abolished the evolution of $\mathrm{CO}_{2}$ from meso-tartaric acid (Fig. 2). This was accompanied by the accumulation of large quantities of $\beta$-ketoacid, as shown by the large amount of $\mathrm{CO}_{2}$ evolved upon addition of 4-aminoantipyrine to the reaction mixture after $15 \mathrm{~min}$. of incubation; in the absence of EDTA, the accumulation of $\beta$-ketoacid was negligible (Fig. 2). The 2-4-dinitrophenyl hydrazones of the ketoacids accumulating from meso-tartrate in the presence and absence of EDTA were prepared and subjected to chromatographic analysis. The reaction mixture without EDTA yielded only the 2-4-dinitrophenylhydrazone of pyruvic acid; the reaction mixture with EDTA yielded also a second 2,4-dinitrophenylhydrazone, chromatographically indistinguishable from the 2,4-dinitrophenylhydrazone of oxaloacetic acid. Furthermore, analysis by the method of Friedemann \& Haugen showed that in the extract treated with EDTA, the total quantity of ketoacid was almost double the quantity of pyruvic acid (Table 2 ).

\section{Table 2. The accumulation of ketoacids from meso-tartaric acid in the presence and absence of $E D T A$}

The incubation mixtures contained : $20 \mu$ mole meso-tartaric acid; $0.5 \mathrm{ml}$. of meso 1 extract; and phosphate buffer $(0.02 \mathrm{M}, \mathrm{pH} \mathrm{7.2)}$ to make $1.5 \mathrm{ml}$. The reactions were stopped by the addition of $1.5 \mathrm{ml}$. of $10 \%$ trichloroacetic acid after $15 \mathrm{~min}$. of incubation at $30^{\circ}$. Pyruvate was estimated in the deproteinized supernatant by the method of Friedman \& Haugen (1943) in xylene extracts. For total ketoacid estimation, ethyl acetate was used as solvent.

\begin{tabular}{|c|c|c|}
\hline Treatment & $\begin{array}{c}\text { Pyruvate } \\
(\mu \text { mole })\end{array}$ & $\begin{array}{c}\text { Total } \\
\text { ketoacids } \\
(\mu \text { mole })\end{array}$ \\
\hline No EDTA added & $18 \cdot 0$ & $17 \cdot 4$ \\
\hline $10 \mu$ mole EDTA & $6 \cdot 9$ & $11 \cdot 05$ \\
\hline
\end{tabular}


By similar methods, the extract from strain $d 15$ was shown to produce oxaloacetic acid from $d$-tartaric acid in the presence of 0.04 M EDTA. The extract from strain $l 1$, however, did not decompose tartaric acid in the presence of 0.04 M-EDTA. With lower concentrations of EDTA (0.004 M), the reaction proceeded and a small accumulation of oxalacetic acid was demonstrated.

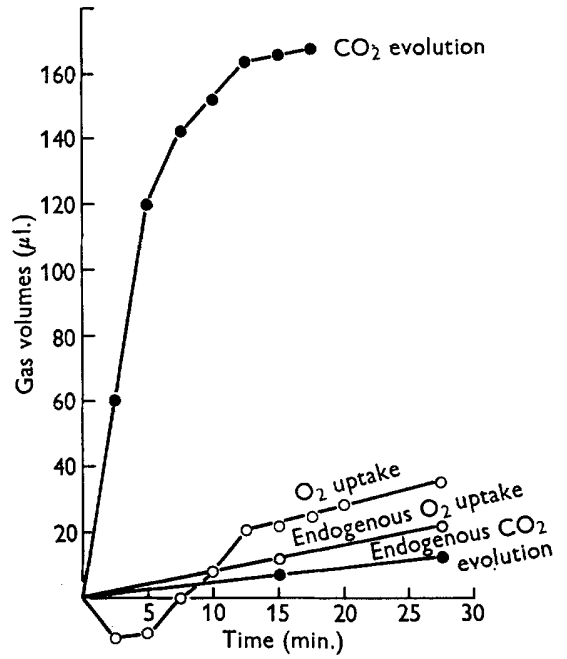

Fig. 1

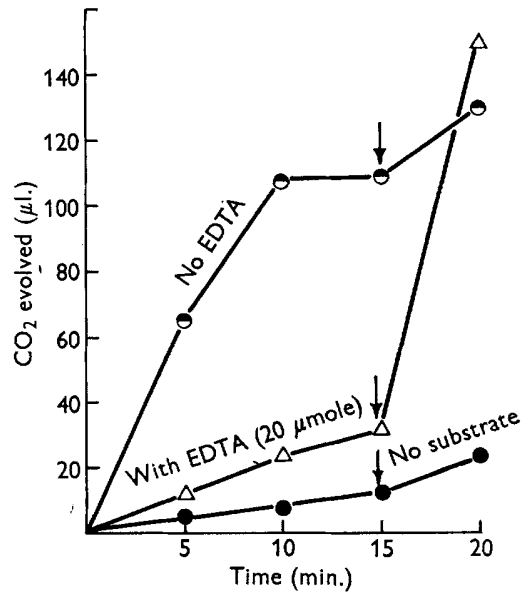

Fig. 2

Fig. 1. $\mathrm{O}_{2}$ uptake and $\mathrm{CO}_{2}$ evolution from meso-tartaric acid by an extract of strain meso 1 . Incubation mixture contained: $5 \mu$ mole neutralized meso-tartrate; $0.5 \mathrm{ml}$. meso 1 extract; phosphate buffer $(0.02 \mathrm{M}, \mathrm{pH} 7 \cdot 2)$ to make $2 \mathrm{ml}$. Temperature $30^{\circ}$.

Fig. 2. Effect of EDTA on $\mathrm{CO}_{2}$ evolution and $\beta$-ketoacid accumulation from meso-tartaric acid by an extract from strain meso 1 . Incubation mixtures contained: $5 \mu$ mole mesotartaric acid; $0.5 \mathrm{ml}$. meso 1 extract; phosphate buffer $(0.02 \mathrm{M}, \mathrm{pH} 7.2)$ to make $1.8 \mathrm{ml}$. Temperature $30^{\circ}$. At points indicated by arrows, the incubation mixture was acidified with $0 \cdot 1 \mathrm{ml}$. $\mathrm{N}$-acetic acid and $0 \cdot 4 \mathrm{ml}$. 4-aminoantipyrine $(0 \cdot 1 \mathrm{M})$ was added.

It is accordingly evident that the decomposition of the three isomers of tartaric acid involves in each case a specific dehydration to oxaloacetic acid (probably the enol form), followed by decarboxylation of the oxaloacetic acid to pyruvic acid. Thus only the initial step in the metabolism of the tartaric acids is biochemically specific and unique; with the formation of oxaloacetic acid and pyruvic acid, the main pathways of intermediary metabolism are attained. We shall term the specific enzymes involved tartaric acid dehydrases.

\section{The development of assays for the tartaric acid dehydrases}

Oxaloacetic acid can be estimated spectrophotometrically by its light absorption at $280 \mathrm{~m} \mu$. Since it accumulates when meso-tartaric acid is enzymically attacked in the presence of EDTA, a spectrophotometric assay which measures the accumulation of oxaloacetic acid directly was developed. Fig. 3 shows the accumulation of oxaloacetic acid in the presence and in the absence of EDTA (10 $\mu$ mole) as estimated from optical density changes at $280 \mathrm{~m} \mu$. 
In the absence of EDTA, there was an initial rapid formation of oxaloacetic acid from meso-tartaric acid, followed by a decline. The maximal quantity of oxaloacetic acid accumulated from $5 \mu$ mole meso-tartrate was less than $2 \mu$ mole. In the presence of EDTA, oxaloacetic acid accumulated steadily, more than $4 \mu$ mole being formed from $5 \mu$ mole meso-tartrate, and more than $8 \mu$ mole from $10 \mu$ mole meso-tartrate. At the points indicated by arrows in

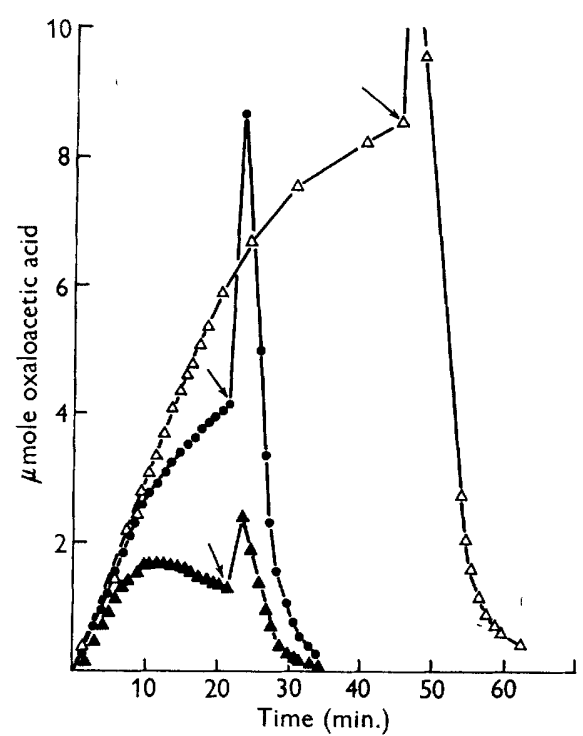

Fig. 3

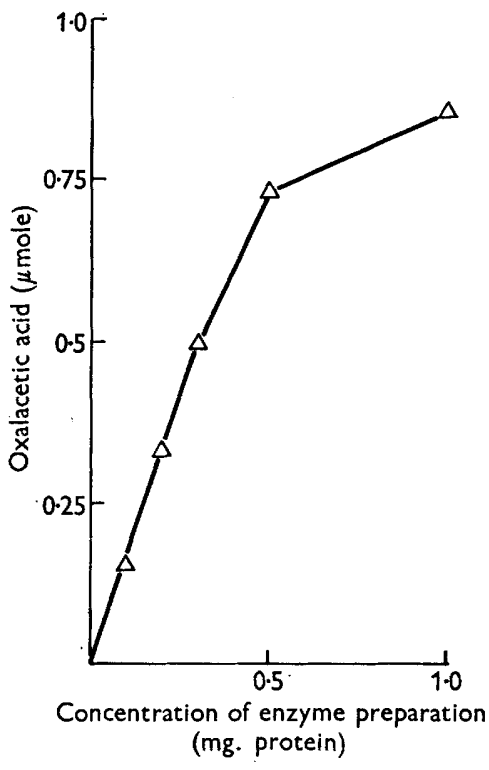

Fig. 4

Fig. 3. Effect of EDTA on the accumulation of oxaloacetic acid from meso-tartaric acid by an extract from strain meso 1. An incubation mixture contained $0.05 \mathrm{ml}$. meso 1 extract and phosphate buffer $(0.02 \mathrm{M}, \mathrm{pH} 7 \cdot 2)$ to make $2.5 \mathrm{ml}$., $\Delta-\Delta, 5 \mu$ mole meso-tartrate; -5 , 5 mole meso-tartrate $+10 \mu$ mole EDTA; $\triangle-\triangle, 10 \mu$ mole meso-tartrate $+10 \mu$ mole EDTA. The accumulation of oxaloacetic acid was followed at $280 \mathrm{~m} \mu$. in the Beckman spectrophotometer. $\mathrm{Mg}^{++}(10 \mu$ mole $)$ was added at points indicated by arrows.

Fig. 4. The effect of enzyme concentration on the rate of accumulation of oxaloacetic acid from meso-tartaric acid. Incubation mixtures containing $10 \mu \mathrm{mole}$ of neutralized meso-tartaric acid, $10 \mu$ mole EDTA, varying amounts of meso 1 extract and phosphate buffer $(0.02 \mathrm{M}, \mathrm{pH} \mathrm{7.2)}$ were tested for differences in the rate of accumulation of oxaloacetic acid, as measured in the Beckman spectrophotometer at $280 \mathrm{~m} \mu$.

Fig. 3, $10 \mu$ mole of $\mathrm{MgCl}_{2}$ were added to the reaction mixtures. In each case there was an immediate rapid rise in optical density, a phenomenon which is also observed when $\mathrm{Mg}^{++}$is added to neutral aqueous solutions of pure oxaloacetic acid. The explanation for this is unknown, but it possibly reflects formation of a magnesium-complex with oxaloacetic acid. Following this initial increase in optical density, the optical density of the reaction mixture fell rapidly to a negligible value, reflecting the decarboxylation of oxaloacetic acid to pyruvic acid. 
From Fig. 3, it can be seen that with a given quantity of enzyme, the initial rate of accumulation of oxaloacetic acid in the presence of EDTA was virtually identical for 5 and $10 \mu$ mole meso-tartrate. The effect of enzyme concentration on the initial rate of formation of oxaloacetic acid in the presence of EDTA is shown in Fig. 4; within limits, there is a linear relationship between enzyme concentration and activity. Hence it is evident that the spectrophotometric method provides a convenient continuous assay procedure for the mesotartaric acid dehydrase in the presence of EDTA. With $d$-tartaric acid dehydrase, the same assay method can be used, although the accumulation of oxaloacetic acid is never so complete, and the rate of its formation starts to

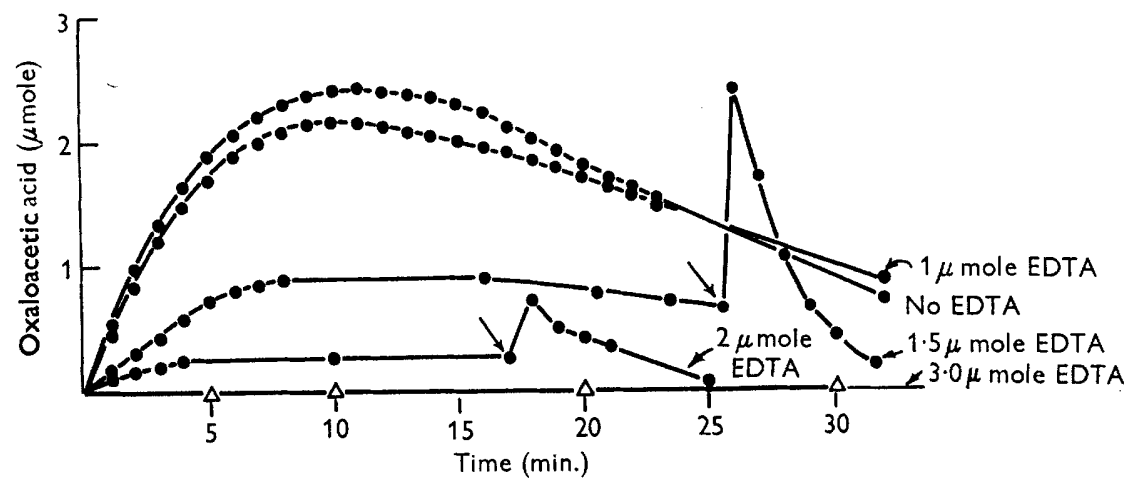

Fig. 5. Effect of EDTA on $l$-tartrate dehydrase. Incubation mixtures contained : 0.04 ml. extract of strain $l 1$ grown on $l$-tartrate medium; $10 \mu$ mole $l$-tartrate; different amounts (0 to $3 \mu$ mole) of EDTA; phosphate buffer $(0.02 \mathrm{M}, \mathrm{pH} \mathrm{7.2})$ to make $2.5 \mathrm{ml}$. At times indicated by arrows, $10 \mu$ mole of $\mathrm{Mg}^{++}$were added. Accumulation of oxaloacetic acid was followed in the Beckman spectrophotometer at $280 \mathrm{~m} \mu$.

decline at an earlier point in the reaction. Although the accumulation of oxaloacetic acid from $l$-tartaric acid in the presence of the specific dehydrase can be demonstrated, this dehydrase is itself strongly inhibited by EDTA (Fig. 5). The assay of this enzyme can be made, although somewhat imprecisely, by measuring the initial rate of accumulation of oxaloacetic acid in a reaction system without EDTA.

\section{General properties of the tartaric acid dehydrases}

The stereospecificity of the three tartaric acid dehydrases is absolute: each enzyme is capable of attacking only one isomer of tartaric acid. Furthermore, no other substrates for any of these enzymes have been found. The reactions catalysed by the meso- and $d$-tartaric acid dehydrases appear to be essentially irreversible: when the enzymes preparations were incubated with oxaloacetic acid, the decrease in optical density at $280 \mathrm{~m} \mu$. was not greater than that observed in controls containing oxaloacetic acid alone. The reversibility of the reaction catalysed by the $l$-tartaric acid dehydrase cannot be tested in this way, since the decarboxylation of oxaloacetic acid cannot be blocked by EDTA treatment. 
Experiments with the dehydrases were usually conducted at $\mathrm{pH} \mathbf{7 \cdot 2}$ in phosphate buffer. The effect of $\mathrm{pH}$ value on the activity of the meso-tartaric acid dehydrase was measured; there was a broad plateau between $\mathrm{pH} 6.9$ and $8 \cdot 5$, with a rapid decline of activity on both sides.

Attempts to purify the meso dehydrase have so far failed. Dialysis and ammonium sulphate precipitation both caused great loss of activity, somewhat decreased by the presence of EDTA. Nevertheless, in crude extracts the meso dehydrase is stable for several weeks when kept frozen. The $d$-and $l$-tartaric acid dehydrases, on the other hand, lost considerable activity when stored for a few days in the frozen state.

\section{Table 3. Patterns of inhibition of the tartaric acid dehydrases by sterically related compounds}

The incubation mixtures contained: $10 \mu$ mole of specific substrate, $10 \mu$ mole of the listed compound, and phosphate buffer $(0.02 \mathrm{M}, \mathrm{pH} 7.2)$ to make $2.5 \mathrm{ml}$. In the cases of the meso dehydrase and the $d$ dehydrase, $10 \mu$ mole of EDTA were also added. The rate of accumulation of oxaloacetic acid was estimated at $280 \mathrm{~m} \mu$. by the Beckman spectrophotometer, in the presence and absence of the compounds tested as inhibitors.

$\%$ inhibition at an equimolar ratio to substrate

$\quad$ Inhibitor
meso-Tartaric acid
$d$-Tartaric acid
$l$-Tartaric acid
Citraconic acid
$d, l$-Citromalic acid
Itaconic acid
$d, l$-trans-Epoxysuccinic acid
$d, l$-cis-Epoxysuccinic acid
Tartronic acid
$d$-Malic acid
$l$-Malic acid

$\begin{array}{ccc}\text { Meso dehydrase } & d \text { dehydrase } & l \text { dehydrase } \\ -74 & 54 & 93 \\ 0 & - & 35 \\ 0 & 0 & - \\ 0 & - & - \\ 0 & - & - \\ 16 & - & - \\ 87 & 56 & 16 \\ 42 & 0 & - \\ 74 & 66 & - \\ 0 & 72 & -\end{array}$

Inhibition pattern. In addition to their stereospecificity with respect tc substrate, the three tartaric acid dehydrases showed characteristic individual patterns of inhibition by compounds sterically related to the substrates (see Table 3). The data were obtained by determining the decrease in the rate of oxaloacetic acid accumulation which occurred when each of the listed inhibitors was added to the enzyme preparation in the presence of an equimolar quantity of the specific substrate. It can be seen that in some cases the degree of inhibition was exceedingly severe: for example, the meso dehydrase was inhibited to the extent of at least $74 \%$ by $d$-tartaric, $d$-malic and $d, l$-cis-epoxysuccinic acids, and the $l$-tartaric acid dehydrase was inhibited to an extent of $85 \%$ or more by meso-tartaric acid and $d, l$-trans-epoxysuccinic acid. It seems likely that these inhibitors act competitively, although this has been definitely shown only for the inhibition of the meso-tartaric acid dehydrase by $d$-tartaric and tartronic acids. 


\section{DISCUSSION}

The enzymic dehydration of tartaric acid to oxaloacetic acid, first established by Krampitz \& Lynen (1956) for the $d$-isomer, occurs also with the meso- and $l$-isomers, and the attack on all three tartaric acids by bacteria of the genus $\boldsymbol{P}$ seudomonas appears to occur principally by means of stereospecific dehydrases. In the present enzymic study, the existence of such stereospecific dehydrases was established for three strains of Pseudomonas. In addition, however, we have examined extracts from many other Pseudomonas strains which can attack one, two, or all three of the tartaric acids; in most cases oxaloacetic acid is an intermediate. This preliminary survey has shown, however, that at least one additional pathway for the metabolism of $d$-tartaric acid exists in pseudomonads. Extracts from certain $d$-specific strains did not yield oxaloacetic acid from $d$-tartaric acid in the presence of EDTA; instead, small amounts of a ketoacid similar in its properties to glyoxylic acid accumulated.

The patterns of inhibition observed with the three tartaric acid dehydrases can be interpreted, at least in part, in steric terms. We shall consider first the inhibitory interactions between the three tartaric acids. meso-Tartaric acid, the internally compensated molecular form, can be regarded as containing both the $d$ and the $l$ configurations. The fact that the meso dehydrase is strongly inhibited by the $d$-isomer of tartaric acid, but not at all by the $l$-isomer, suggests that it is that portion of the meso-tartaric acid molecule that contains the $d$-configuration which is actually bound to the meso dehydrase. The $d$ dehydrase is inhibited by the meso-isomer but not by the $l$-isomer, while the $l$ dehydrase is inhibited by the meso-isomer but not by the $d$-isomer. These facts suggest that the combining sites of the tartrate dehydrases are specific for the enantiomorphic configuration shown by their respective substrates; and, further, that only one of the asymmetric carbons of the substrate is involved in the enzyme-substrate combination.

These interpretations of the minimal steric configurations necessary for binding by the three dehydrases also fit the observed inhibitions with other substances sterically related to the tartaric acids. Thus $d$-malic acid inhibits the meso dehydrase and the $d$ dehydrase. Tartronic acid, which has the essential structure of the postulated combining region of the substrates, but lacks asymmetry, also inhibits the meso and $d$ dehydrases. Lastly, there are the trans- and cis-epoxysuccinic acids to be considered. The $d l$ - cis form strongly inhibits only the meso dehydrase, while the $d l$-trans form strongly inhibits the $d$ and $l$ dehydrases, but only slightly the meso dehydrase. Here it seems evident that geometrical configurations also play a role in the inhibitory process; until the enantiomorphs of each geometrical isomer have been tested separately, a detailed analysis in steric terms cannot be made.

The work described in this paper was performed while the author held a Judah L. Magnes Fellowship, during leave from the Laboratory for Microbiological Chemistry, Hebrew University, Hadassah Medical School, Jerusalem, Israel. It was aided by a grant from the cancer research funds of the University of California. 


\section{REFERENCES}

Akamatsu, S. (1950). $\beta$-Carboxylase model. J. Biochem., Tokyo, 37, 65.

Barker, H. A. (1936). On the fermentation of some dibasic $\mathrm{C}_{4}$-acids by Aerobacter aerogenes. Proc. Acad. Sci. Amst. 39, 674.

Buchanan, C. (1951). Configurational notation of the tartaric acids. Nature, Lond. $167,689$.

CAHN, R. S. \& INGold, C. K. (1951). Specification of configuration about quadricovalent asymmetric atoms. J. chem. Soc. p. 612.

Cammarata, P. S. \& Cohen, P. P. (1951). Spectrophotometric measurement of transamination reactions. J. biol. Chem. 193, 45.

Cavallini, D., Frontali, N. \& Toshi, G. (1949). Keto acid content of human blood and urine. Nature, Lond. 164, 792.

Friedman, T. E. \& Haugen, G. E. (1943). Pyruvic Acid. II. The determination of keto acids in blood and urine. J. biol. Chem. 147, 415.

Green, D. E., Leloir, L. F. \& Nocito, V. (1945). Transaminases. J. biol. Chem. 161, 559.

Krampitz, L. O. \& Lynen, F. (1956). Formation of oxalacetate from d-tartrate. Fed. Proc. 15, 292.

Kun, E. (1956). Enzymatic mechanism of oxidation of tartrate. J. biol. Chem. 221, 223.

Kun, E. \& Davies, D. D. (1956). Enzymatic components of the tartrate oxidizing system of beef heart mitochondria. Fed. Proc. 15, 294.

Kun, E. \& Hernandez, M. G. (1956). The oxidation of tartaric acid by an enzyme system of mitochondria. J. biol. Chem. 218, 201.

Martin, R. W. \& Foster, J. W. (1955). Production of trans- $l$-epoxysuccinic acid by fungi and its microbiological conversion to meso-tartaric acid. J. Bact. 70, 405.

Matchett, J. R., Legault, R. R., Nimmo, C. C. \& Notter, G. K. (1944). Tartrates from grape wastes. Industr. Engng Chem. (Industr.), 36, 851.

Mercer, W. A. \& VAUghn, R. H. (1951). The characteristics of some thermophilic tartrate-fermenting anaerobes. J. Bact. 62, 27.

Nomura, M. \& Sakaguchi, K. (1955). The decomposition of L (+) tartrate by micro-organisms. J. gen. Appl. Microbiol. (Japan), 1, 77.

Pasteur, L. (1860). Note relative à Penicillium glaucum et à la dissymétrie moléculaire des produits organiques naturels. C.R. Acad. Sci., Paris, 51, 298.

Pasteur, L. (1863). Nouvel exemple de fermentation déterminée par des animalcules infusoires pouvant vivre sans gaz oxygène libre et en dehors de tout contact avec l'air de l'atmosphère. C.R. Acad. Sci., Paris, 56, 416.

Rivière, J. W. M. LA (1956). Intermediate products in tartrate decomposition by cell-free extracts of Pseudomonas putida under anaerobic conditions. Biochim. biophys. Acta, 21, 190.

Scholefield, P. G. (1955). The oxidation of malic and meso-tartaric acids in pigeon-liver extracts. Biochem. J. 59, 177.

Shilo, M. \& STANiER, R. Y. (1957). The utilization of the tartaric acids by pseudomonads. J. gen. Microbiol. 16, 482.

Sistrom, W. R. \& Stanier, R. Y. (1953). The mechanism of catechol oxidation by Mycobacterium butyricum. J. Bact. 66, 404.

Stadtman, T. C., Vaughn, R. H. \& Marsh, G. L. (1945). Decomposition of tartrates by some common fungi. J. Bact. 50, 691.

TABachnick, J. \& VAUGhN, R. H. (1948). Characteristics of tartrate-fermenting species of Clostridium. J. Bact. 56, 435.

Vaughn, R. H., Marih, G. L., Stadtman, T. C. \& Cantino, B. C. (1946). Decomposition of tartrates by the coliform bacteria. J. Bact. 52, 311.

\section{(Received 24 October 1956)}

